\title{
Étude numérique d'un modèle réduit de remblai sur argile molle
}

\section{Y. AKOU}

J.-P. MAGNAN

Laboratoire central des Ponts et Chaussées, 58, boulevard Lefebvre, 75732 Paris Cedex 15

Des calcuis en éléments finnis d'une expérience d'élargissement de remblai sur argile molle en centrifugeuse ont été exécutés avec le module CSNL de CÉSAR-LCPC. Ces calculs sont de type élastoplastique couplé, avec une loi de comportement élastoplastique anisotrope avec écrouissage pour l'argile.

L'article décrit la détermination des paramètres de calcul, le maillage et la loi de chargement, et les hypothèses faites sur les sols et le remblai ; puis il présente les résultats du calcul et les compare aux mesures, d'une part, et aux calculs exécutés par les méthodes classiques (abaques d'élasticité à court terme ; méthode œedométrique pour les tassements de consolidation), d'autre part. Les tassements et pressions interstitielles observés sont assez bien reproduits par le calcul.

Mots clés : éléments finis (méthode), CESAR-LCPC (code d'éléments fĩnis), modèle (rhéologique, Mélanie), modélisation-élargíssement de remblai, sol mou.

\section{Numerical analysis of a small scale model of embankment on soft clay}

Finite element analyses of the centrifuged model of a wiciened embankment on soft clay have been performed using the finite element program CSNL of CESAR-LCPC. Coupled elastoplastic analyses were performed, using an anisotropic strain-hardening elastoplastic model for the clay behaviour:

Design parameters for the foundation soil and the fill are first described, together with the finite element mesh and the loading procedure used in the analyses. The numerical results are compared to the measured settlements and pore pressures and to the results of classical methods used to estimate the immediate undrained settlements (elastic analysis) and consolidation settiements (based on the oedometer test). Both settlements and pore pressures were satisfactorily predicted by the numerical analyses.

Key words : finite element method, stress-strain model, modelling, embankment widening, soft soil. 


\section{NOTATIONS}

$C_{c}$ : indice de compression.

$\mathrm{C}_{\mathrm{S}}$ : indice de gonflement.

$\mathrm{C}_{a}$ : coefficient de compression secondaire

$\mathrm{C}_{\alpha c}$ : indice de fluage.

E : module d'Young.

$\mathrm{E}_{h}^{\prime}$ : module de déformation horizontal.

$E_{y}^{\prime}$ : module de déformation vertical.

$\mathrm{G}_{v h}^{\prime}:$ module de cisaillement dans le plan vertical.

$K_{0}^{n c} \quad$ : coefficient de pression des terres au repos dans le domaine normalement consolidé.

$\mathrm{K}_{0}^{\circ} \quad$ : coefficient de pression des terres au repos dans le domaine surconsolidé.

$R_{c \text { : }} \quad$ rapport de surconsolidation.

Tolc : tolérance sur la convergence du critère de plasticité.

$a_{w}$ : coefficient de compressibilité de l'eau.

$c^{\prime} \quad$ : cohésion effective.

$\mathrm{c}_{u}$ : cohésion non drainée.

e : indice des vides.

$e_{0} \quad$ : indice des vides initial.

n : porosité.

$\mathrm{k}_{\mathrm{h}}$; coefficient de perméabilité horizontale.

$k_{v} \quad$ : coefficient de perméabilité verticale.

$\mathrm{S}_{\mathrm{p}} \quad$ : paramètre d'écrouissage du modèle Mélanie.

t : temps.

$\Delta t \quad$ : pas de temps.

$\gamma_{\mathrm{w}}$ : poids volumique de l'eau.

$\gamma$ : poids volumique du sol.

$\eta \quad$ : coefficient de normalité (loi d'écoulement plastique du modèle Mélanie).

$\varphi^{*} \quad$ : angle de frottement interne.

$\lambda \quad$ : pente de la courbe vierge isotrope $(e-$ Ins').

$v \quad$ : coefficient de Poisson.

$v_{\text {hh }}$ : coefficient de Poisson dans le plan horizontal.

$v_{v i}^{\prime}$ : coefficient de Poisson dans le plan vertical.

$\sigma_{p}^{\prime} \quad$ : pression de préconsolidation.

$\sigma^{\prime} \quad$ : contrainte effective verticale.

$\sigma_{\mathrm{yf}}:$ contrainte effective verticale finale.

$\sigma_{v o}:$ contrainte effective verticale initiale.

$\Psi$ : angle de dilatance.

\section{Introduction}

L'étude présentée dans cet article a été réalisée dans le cadre d'un programme de recherche sur l'élargissement des remblais sur sols mous entrepris au début des années 1990 et comportant des expérimentations en vraie grandeur et en centrifugeuse et des analyses théoriques. L'objectif de ces recherches était de préciser les méthodes de calcul des tassements du sol lors de l'élargissement de remblais existants, en allant audelà de la méthode classique de calcul des tassements par la méthode cedométrique, qui distingue le calcul des variations de contraintes verticales en élasticité isotrope (abaque d'Osterberg) et le calcul des amplitudes de tassements d'après les courbes de compressibilité œdométrique.

Des calculs en éléments finis et des calculs "manuels » classiques par abaques ont été exécutés sur les modèles testés en centrifugeuse, afin de comparer leurs prévisions avec les résultats des mesures de tassements et de pressions interstitielles effectuées en cours d'essai et de contribuer ainsi à la compréhension des phénomènes observés sur les modèles. Les calculs en éléments finis, exécutés avec le module CSNL de CESAR-LCPC, tiennent compte de la montée en accélération au début de l'essai et de la consolidation du sol au cours du temps.

Après un rappel du déroulement de l'essai en centrifugeuse, cet article présente les hypothèses et les résultats du calcul en éléments finis, puis les compare avec les mesures de tassements et de pressions interstitielles effectuées en cours d'essai (Akou, 1995) et avec les résultats des calculs par abaques.

\section{2}

\section{Données expérimentales}

La modélisation numérique porte sur l'élargissement en cours de centrifugation d'un remblai édifié sur un massif d'argile « Speswhite » reconstituée. Le remblai initial et le remblai qui lui est accolé sont constitués de sable de Fontainebleau. Le remblai adjacent a une largeur en crête égale à une fois et demie celle du remblai initial. Cet essai porte le numéro 5 dans l'étude expérimentale (Akou, 1995; Akou et al., 1997)

Le modèle centrifugé était équipé de 11 capteurs de tassement de surface (numérotés de 1 à 11), de cinq capteurs de pression interstitielle et de trois inclinomètres pour la mesure des déplacements horizontaux (Fig. 1). Les mesures de déplacements latéraux n'ont pu ètre interprétée de façon cohérente et ne sont pas utilisées ici.
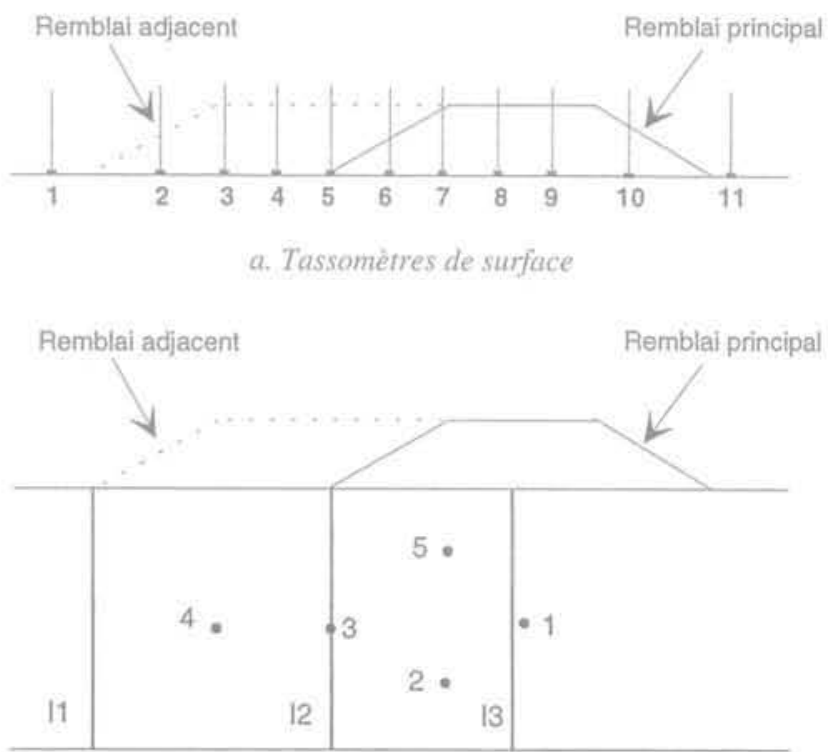

b. Piézomètres et tubes inclinométriques

FG. I Instrumentation du modèle centrifugé. Instrumentation of the centrifuged model. a Surface settlement gauges, b. Piezometers and inclinometer tubes. 
Les dimensions du modèle sont les suivantes :

- épaisseur de la couche d'argile

$15,7 \mathrm{~cm}$;

- largeur à la base du remblai initial

$32,9 \mathrm{~cm}$;

- largeur en crête du remblai initial

$12,9 \mathrm{~cm}$;

- largeur à la base du remblai adjacent $20 \mathrm{~cm}$;

- largeur en tête du remblai adjacent $20 \mathrm{~cm}$.

L'argile repose sur une couche de sable drainant. L'argile est saturée et le niveau de la nappe est maintenu au niveau de la surface de l'argile pendant l'essai.

\section{3}

\section{Programme de calcul en éléments finis}

L'analyse en éléments finis de la consolidation du massif d'argile du modèle a été effectuée en utilisant le module CSNL de CÉSAR-LCPC (Humbert, 1989). Successeur du programme de calcul ROSALIE-Groupe 9, développé au LCPC au cours des années 1970, ce module permet la résolution des problèmes de consolidation en conditions uni-, bi- et tridimensionnelles, en utilisant des modèles élastoplastiques tels que CamClay (Dang et Magnan, 1977), Mélanie (Mouratidis et Magnan, 1983 ; Lepidas et Magnan, 1990 ; Kattan, 1990 ; Akou, 1995), ou les autres modèles de comportement élastoplastiques disponibles dans CÉSAR-LCPC.

\section{4}

\section{Modèle de comportement de l'argile}

Le modèle développé au LCPC pour représenter le comportement des argiles naturelles dans les calculs en éléments finis (Mélanie) est un modèle élastoplastique anisotrope avec écrouissage dérivé du modèle Cam-clay modifié. La figure 1 montre les caractéristiques principales de sa courbe d'état limite (critère de plasticité). L'écrouissage est déduit des courbes de compressibilité œdométrique. A l'intérieur du domaine élastique (intérieur de l'ellipse), le sol est supposé élastique linéaire anisotrope. Sur la frontière du domaine élastique, l'écoulement plastique s'effectue selon une loi non associée.

Les paramètres du modèle sont déduits des caractéristiques physiques et mécaniques du sol mesurées au cours d'études géotechniques courantes, à l'exception des paramètres d'élasticité linéaire anisotrope. Ces paramètres, au nombre de cinq $\left(E_{v}^{\prime}, E^{\prime}, v_{h h}^{\prime}, v_{v}^{\prime}, G_{v}^{\prime}\right)$, peuvent être déterminés au moyen d'essais triaxiaux avec consolidation anisotrope sur des éprouvettes d'orientations différentes (Piyal et Magnan, 1984). Toutefois, compte tenu de la difficulté de réalisation de ces essais, les valeurs de ces paramètres sont souvent choisies d'après les valeurs trouvées sur d'autres sites, c'est-à-dire que l'on fixe a priori les valeurs de $n^{\prime}=E_{n}^{\prime} / E_{v}^{\prime}, m^{\prime}=G_{v y}^{\prime} / E_{v}^{\prime}, v_{n h}^{\prime}$ et $v_{h h}^{\prime}$ et qu'il suffit alors de calculer le module $\mathrm{E}^{\prime}$ à partir de la courbe oedométrique (courbe de recompression dans le domaine surconsolidé), au moyen de la relation:

$$
\begin{gathered}
E_{\mathrm{v}}^{\prime}=\frac{\Delta \sigma_{\mathrm{v}}}{\varepsilon_{\mathrm{v}}}\left[1-\frac{2 v_{\mathrm{vh}}{ }^{2} \mathrm{n}}{1-v_{\mathrm{hh}}^{\prime}}\right]= \\
\frac{1+\mathrm{e}_{\mathrm{b}}}{\mathrm{C}_{\mathrm{s}}} \cdot \frac{\sigma_{\mathrm{vf}}-\sigma_{\mathrm{vo}}}{\lg \left(\sigma_{\mathrm{vf}} / \sigma_{\mathrm{vo}}\right)} \cdot\left[1-\frac{2 v_{\mathrm{vh}}{ }^{2} \mathrm{n}}{1-v_{\mathrm{hh}}}\right]
\end{gathered}
$$

Cette relation traduit la linéarisation de la relation semilogarithmique $\Delta \mathrm{e}=\mathrm{C}_{\Delta} \Delta / g \sigma_{v}^{\prime}$ entre une contrainte initiale $\sigma_{\mathrm{vo}}^{\prime}$ et une contrainte finale $\sigma_{\mathrm{v}}^{\prime}$. Lorsque le sol est dans un état initial proche de la pression de préconsolidation, on calcule en général le module entre la contrainte verticale initiale $\sigma_{v o}^{\prime}$ et la pression de préconsolidation $\sigma_{p}^{\prime}$

La forme des courbes d'état limite, qui sont homothétiques par rapport à l'origine des axes (Os', Ot), dépend du coefficient de pression des terres au repos du sol normalement consolidé $K_{0}^{n}$, qui définit l'angle entre le grand-axe de l'ellipse et l'axe Os' (Fig. 2). Ce coefficient est en génèral calculé au moyen de l'expression:

$$
\mathrm{K}_{0}^{\mathrm{nc}}=1-\sin \varphi^{\prime}
$$

avec $\varphi^{\prime}$ : angle de frottement interne de l'argile,

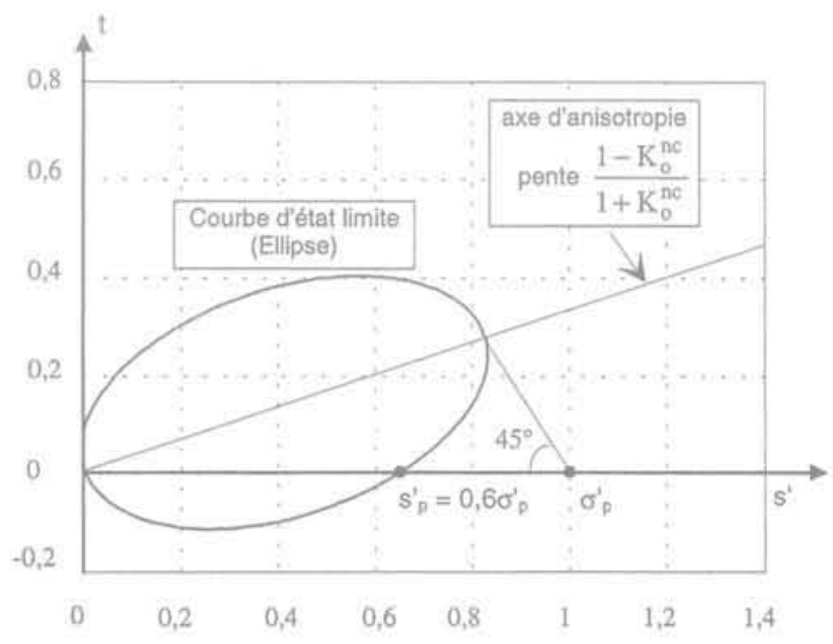

FG.2 Courbe d'état limite pour argile molle naturelle : modèle Mélanie (Lepidas et Magnan, 1990).

Limit state curve for natural soft clays : Mélanie model (Lepidas and Magnan, 1990).

Pour définir l'ellipse correspondant à l'état initial du sol, on doit déterminer la pression de préconsolidation $\sigma^{\prime}$ qui peut ètre déduite d'un essai cedométrique. Lé même essai fournit l'indice de gonflement $C_{s}$ et l'indice de compression $C$ qui servent à calculer le module $\mathrm{E}_{v}^{\prime}$ et la pente $\lambda$ de la courbe vierge isotrope $\left(\mathrm{e}-\ln \mathrm{s}^{\prime}\right)$ :

$$
\lambda=\mathrm{C}_{c} / \ln 10=\mathrm{C}_{\mathrm{c}} / 2,3
$$

Le paramètre d'écrouissage du modèle est la pression s' reliée à la pression de préconsolidation par la relation d'origine expérimentale :

$$
s_{p}=0,6 \sigma_{p}
$$

L'état initial de l'argile est défini par la contrainte verticale effective $\sigma^{\prime}$. I'indice des vides initial $e_{0}$ et le coefficient de pression des terres au repos dans le domaine surconsolidé $\mathrm{K}_{r}^{\circ}$. Ce dernier peut être calculé en utilisant la relation :

$$
\mathrm{K}_{\mathrm{o}}^{o c}=\mathrm{K}_{0}^{\mathrm{nc}} \sqrt{\mathrm{R}_{\mathrm{oc}}}
$$

avec $R_{o c}=\sigma_{p}^{\prime} / \sigma_{v o}^{\prime}$; rapport de surconsolidation du sol.

D'autres paramètres sont nécessaires pour décrire le comportement de l'eau interstitielle et son écoulement à travers les pores du sol. On admet que les pores de l'argile sont remplis d'un liquide interstitiel dont la compressibilité, caractérisée par le coefficient de compressibilité a 
dépend du degré de saturation $S$ de l'argile. L'écoulement du liquide est décrit par les coefficients de perméabilité verticale $k_{v}$ et horizontale $k_{h}$. Les paramètres $a_{w}, k_{v}$ et $k_{h}$ peuvent varier au cours du temps en fonction de la pression interstitielle et de l'indice des vides, respectivement.

\section{5}

\section{Propriétés physiques et mécaniques des matériaux pour le calcul}

Les caractéristiques mécaniques et hydrauliques du massif d'argile et des deux remblais ont été déduites des essais de caractérisation de l'argile et du sable réalisés lors de l'étude expérimentale, en particulier d'un essai réalisé en fin d'expérimentation sur l'argile du modèle. Pour calculer le module d'élasticité de l'argile au moyen de la relation (1), on a utilisé les valeurs suivantes des paramètres non mesurés, valeurs issues de l'étude de l'argile molle organique de Cubzac-les-Ponts :

$$
\begin{array}{ll}
v_{\text {hh }}^{\prime}=0,1 & n^{\prime}=E_{h}^{\prime} / E_{v}^{\prime}=0,625 \\
v_{v h}^{\prime}=0,25 & m^{\prime}=G_{v h}^{\prime} / E_{v}^{\prime}=0,450
\end{array}
$$

Une fois déterminé ces paramètres, il reste à définir la valeur du module E', au moyen de la relation [1], c'est-àdire à choisir l'intervalle de contraintes sur lequel on linéarisera la courbe de compressibilité semi-logarithmique.

Sur ce point, les essais en centrifugeuse comportent une phase initiale au cours de laquelle on passe d'une contrainte très faible à la contrainte effective considérée comme initiale sur le terrain $\left(\sigma_{\text {vo }}^{\prime}\right)$, puis une phase où l'on passe de cette contrainte effective initiale à la contrainte de préconsolidation, comme sur un site réel. Pour permettre la modélisation des différentes phases de l'essai, y compris la phase de reconsolidation sous le poids propre du sol, nous avons choisi de déterminer deux jeux de paramètres d'élasticité anisotrope: - un jeu de paramètres pour la phase initiale, au cours de laquelle l'état de contrainte passe d'une valeur très faible à sa valeur ( réelle ») in situ ;

- un jeu de paramètres pour représenter ce qui se passe lors de la construction des remblais et de la consolidation qui suit.

Pour trouver le premier jeu de modules, nous avons linéarisé la déformation entre l'état de contraintes du modèle sous $1 \mathrm{~g}$ et l'état de contrainte final du modèle sous $70 \mathrm{~g}$. Pour trouver le second jeu de modules, nous avons linéarisé la déformation entre l'état initial sous $70 \mathrm{~g}$ et la pression de préconsolidation (ou la contrainte effective finale, si le sol reste surconsolidé).

Le coefficient de perméabilité a été également déduit des essais œedométriques. Il vaut $8,4.10^{-10} \mathrm{~m} / \mathrm{s}$ (soit 7,26.10-5 m/jour).

Les propriétés physiques et mécaniques introduites dans les calculs sont résumées dans les tableaux I et II ; les couches sont décrites par référence à la géométrie du prototype, déterminée classiquement avec un facteur d'échelle égal au nombre de g pendant l'essai (dimensions du modèle multipliées par 70).

Notons que les indices de gonflement C. utilisés pour calculer les modules ont été diminués (d'un facteur 2,5) par rapport aux valeurs tirées des essais $\left(\mathrm{C}_{\mathrm{s}}=\right.$ $0,04$ au lieu de 0,1$)$. Cette correction a pour effet de ramener le rapport $\mathrm{C}_{\mathcal{L}} \mathrm{C}_{\mathrm{s}}$ à des valeurs plus classiques (entre 10 et 15). Tous les modules utilisés dans les calculs ont été déterminés dans le cas oủ le sol reste surconsolidé en cours d'essai.
Pour le sable, un modèle élastoplastique à critère de Mohr-Coulomb a été utilisé, mais le remblai a été maintenu dans un état élastique en jouant sur la cohésion effective.

\section{TABLEAUI Caractéristiques mécaniques et hydrauliques du massif d'argile. Hydraulic and stress-strain characteristics of

\begin{tabular}{|c|c|c|c|c|c|c|}
\hline & \multicolumn{6}{|c|}{ Couches du massif d'argile (échelle du prototype) } \\
\hline$\gamma\left(\mathrm{kN} / \mathrm{m}^{3}\right)$ & 17,46 & 17,46 & 17.46 & 17,46 & 17,46 & 17,46 \\
\hline$v_{n h}^{\prime}$ & 0,1 & 0,1 & 0,1 & 0,1 & 0,1 & 0,1 \\
\hline$v_{\text {th }}^{\prime}$ & 0,25 & 0,25 & 0,25 & 0,25 & 0,25 & 0,25 \\
\hline & & emièr & $\begin{array}{l}\text { lase d: } \\
\text { ératio. }\end{array}$ & $\begin{array}{l}\text { argeme } \\
\text { consoli }\end{array}$ & ion) & \\
\hline$E_{\mathrm{A}}^{\prime}(\mathrm{kPa})$ & 66,8 & 267,22 & 534,45 & 801,67 & 1068,87 & 1068,88 \\
\hline $\mathrm{E}^{\prime},(\mathrm{KPa})$ & 106,9 & 427,55 & 855,1 & 1282,75 & 110,22 & 2137,77 \\
\hline $\mathrm{G}_{\mathrm{m}}^{\prime}(\mathrm{kPa})$ & 48,1 & 192,4 & 384.8 & 576,67 & 769.6 & 962 \\
\hline
\end{tabular} the clay mass.}

\begin{tabular}{|c|c|c|c|c|c|c|}
\hline$E_{n}^{\prime}(\mathrm{kPa})$ & 1349,44 & 2485,46 & 3749,62 & 4948,43 & 6122,32 & 7283,72 \\
\hline$E_{v}^{\prime}(\mathrm{kPa})$ & 2159,11 & 3976,74 & 5999,40 & 7917,49 & 9795,71 & 11653,9 \\
\hline$G_{n t}^{\prime}(\mathrm{kPa})$ & 971,60 & 1789,53 & 2699,73 & 3562,87 & 4408,07 & 5244,28 \\
\hline$\gamma_{w}(\mathrm{kPa})$ & 10 & 10 & 10 & 10 & 10 & 10 \\
\hline$n$ & 0,56 & 0,56 & 0,56 & 0,56 & 0,56 & 0,56 \\
\hline$a_{w}\left(\mathrm{kPa}^{-1}\right)$ & 0 & 0 & 0 & 0 & 0 & 0 \\
\hline$k_{h}(m / j)$ & $7,26,10^{-5}$ & $7,26.10^{\circ}$ & $7.26 .10^{-5}$ & $7,26 \cdot 10^{\text {s }}$ & $7,26.10^{5}$ & $7,26,10^{-5}$ \\
\hline$k_{v}(\mathrm{~m} / \mathrm{j})$ & idem & idem & jdem & fdem & idem & jdem \\
\hline$\lambda$ & 0,217 & 0,217 & 0,217 & 0.217 & 0,217 & 0,217 \\
\hline$e_{b}$ & 1,316 & 1,316 & 1,316 & 1,316 & 1,316 & 1,316 \\
\hline$\sigma_{p}^{\prime}(\mathrm{kPa})$ & 300 & 300 & 300 & 300 & 300 & 300 \\
\hline $\mathrm{K}_{0}^{\infty}$ & 0,642 & 0,642 & 0,642 & 0,642 & 0,642 & 0,642 \\
\hline $\mathrm{K}_{i}^{\mathrm{ni}}$ & 0.642 & 0,642 & 0,642 & 0,642 & 0,642 & 0,642 \\
\hline$\eta$ & 1 & 1 & 1 & 1 & 1 & 1 \\
\hline Tolc & 2000 & 2000 & 2000 & 2000 & 2000 & 2000 \\
\hline
\end{tabular}

Deuxième et troisième phases du chargement (remblais)

Etat de contraintes effectives initiales (première phase)

\begin{tabular}{l|c|c|c|c|c|c}
\hline$\sigma_{\text {vo }}^{\prime}(\mathrm{kPa})$ & 0,05 & 0,213 & 0,426 & 0,639 & 0,852 & 1,066 \\
\hline \multicolumn{7}{c}{$\begin{array}{c}\text { État de contraintes effectives initiales } \\
\text { (deuxième et troisième phases) }\end{array}$} \\
\hline$\sigma_{\text {vo }}^{\prime}(\mathrm{kPa})$ & 3,73 & 14,92 & 29,84 & 44,76 & 59,68 & 74,60 \\
\hline
\end{tabular}

Liste des paramètres du calcul

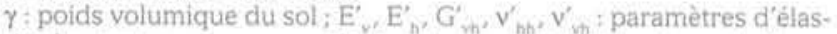
ticité anisotrope $; n$ : porositẻ du sol; $\gamma_{w}$ : poids volumique de l'eau; $a_{w}$ : coefficient de compressibilité de l'eau; $k_{,}, k_{4}$; coefficients de perméabilité verticale et horizontale ; $\lambda$ : indice de compression isotrope; $\mathrm{e}_{0}$ : indice des vides initial; $\mathrm{K}_{\mathrm{o}}^{\mathrm{nc}}$ et $\mathrm{K}_{\mathrm{o}}^{\circ}$ - coefficient de pression des terres au repos, dans le domaine normalement consolidé et dans le domaine surconsolidé, respectivement ; $\eta$ : indice de normalité de la loi d'écoulement, égal à 0 pour une loi associée et 1 pour une loi non associée; Tolc : tolérance de calcul sur le critère de plasticité. 
TABLEAUII Caractéristiques mécaniques et hydrauliques des remblais. Hydraulic and stress-strain characteristics of the fill material.

\begin{tabular}{|c|c|c|c|c|c|c|c|c|c|c|}
\hline$\frac{\gamma}{\left(\mathrm{kN} / \mathrm{m}^{3}\right)}$ & $\begin{array}{c}\mathrm{E} \\
(\mathrm{kPa})\end{array}$ & $\mathrm{v}$ & $\underset{(\mathrm{kPa})}{\gamma}$ & n & $\frac{\mathrm{a}}{\left(\mathrm{kPa}^{-1}\right)}$ & $\underset{(\mathrm{m} a \mathrm{j})}{\mathrm{k}}$ & $\underset{(m / j)}{k_{v}}$ & $\begin{array}{c}\mathrm{c}^{\prime} \\
(\mathrm{kPa})\end{array}$ & $\begin{array}{c}\varphi \\
\text { (degrés) }\end{array}$ & $\begin{array}{c}\Psi \text { ' } \\
\text { (degrés) }\end{array}$ \\
\hline 15,3 & 36000 & 0,35 & 0 & 0,42 & 0 & 15,2 & 15,2 & 500 & 35 & 35 \\
\hline
\end{tabular}

\section{6}

\section{Maillage et conditions aux limites}

Pour effectuer le calcul correspondant à l'essai 5, nous avons utilisé le maillage et les conditions aux limites de la figure 3 . Ce maillage comporte 1712 nœuds et 537 éléments quadrilatères isoparamétriques à 8 nœuds. Ses dimensions sont définies à l'échelle du prototype, au sens usuel des études en centrifugeuse (dimensions du modèle réel multipliées par 70 ). Les conditions aux limites prises en compte dans ce calcul sont les suivantes :

- drainage par les faces inférieure et supérieure du massif de sol ;

- déplacements horizontaux nuls en bas et sur les faces latérales du massif de sol ;

- déplacements verticaux nuls en bas du massif de sol.

L'état initial est caractérisé par une distribution hydrostatique des pressions interstitielles, avec une nappe au niveau de la surface de l'argile, des déplacements horizontaux et verticaux nuls en tous les points du maillage et des contraintes initiales déduites du poids volumique et du coefficient de pression des terres au repos dans le domaine surconsolidé, $\mathrm{K}_{\circ}^{\circ}$. Les remblais ont initialement un poids volumique nul, qui varie par la suite conformément à la loi de chargement.

Le calcul est effectué en trois étapes, correspondant respectivement à la consolidation du sol sous son poids propre, puis à la construction des remblais principal et adjacent. Afin d'éliminer les deux remblais dans la première phase des calculs, bien qu'ils existent dans le maillage, on leur a attribué des modules d'Young et de cisaillement très faibles. Les caractéristiques des éléments des remblais reprennent leurs valeurs réelles dès le début de leur construction.
La montée en accélération de la centrifugeuse, au début de la première phase, est traduite par l'augmentation du poids volumique des éléments représentant le massif d'argile. A l'arrivée à $70 \mathrm{~g}$, le poids volumique est maintenu constant pendant toute la durée de la reconsolidation du sol sous son poids propre. La construction des deux remblais est également modélisée par l'augmentation du poids volumique des éléments constituant le remblai, selon le schéma de chargement représenté sur la figure 4.

Les pas de temps utilisés dans le calcul ont été déterminés d'après la formule proposée par Vermeer et al. (1981) :

$$
\Delta t \geq \frac{\gamma_{w}(\Delta h)^{2}}{6 k_{w} E}
$$

avec :

$\Delta \mathrm{h}$ épaisseur maximale des éléments du maillage :

$\gamma_{w}$ poids volumique de l'eau;

$k_{w}$ coefficient de perméabilité :

E module d'Young.

\section{7}

\section{Comparaison des calculs et des mesures}

\section{1}

\section{Représentation des résultats}

L'analyse des résultats expérimentaux et des résultats des calculs a été effectuée à l'échelle du prototype (dimensions égales à 70 fois celles du modèle) et en uti-

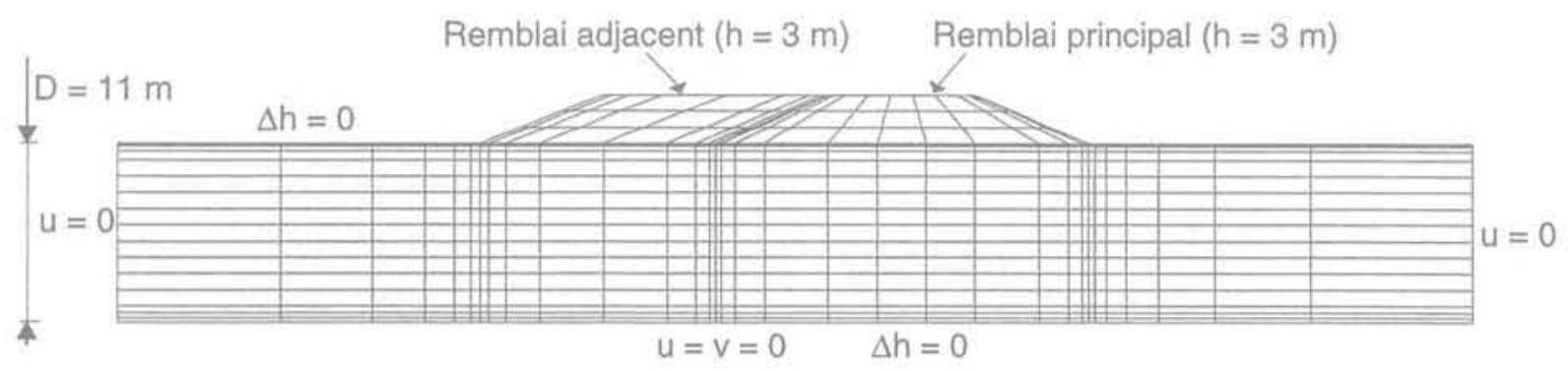

FG. 3 Maillage et conditions aux limites du calcul. FE mesh and boundary conditions. 


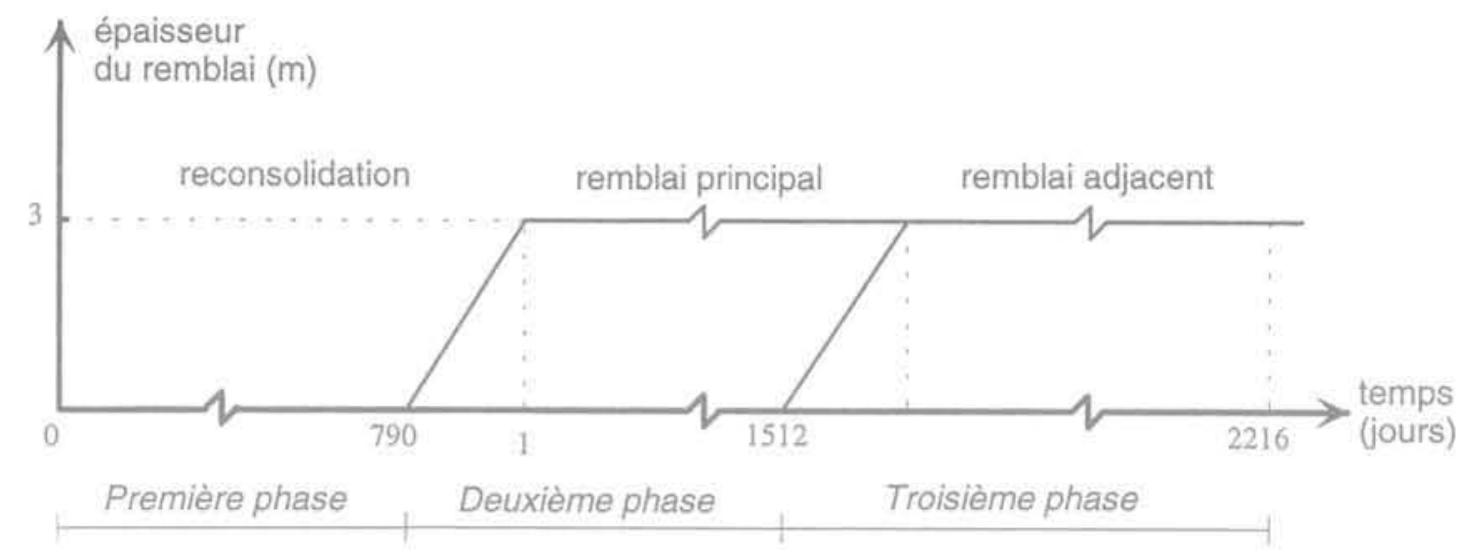

FIG. 4 Augmentation au cours du temps du poids volumique des remblais (épaisseur d'un remblai équivalent de poids volumique fixe).

Phase 1 : reconsolidation de l'argile sous $70 \mathrm{~g}$;

Phase 2 : construction du remblai principal et consolidation :

Phase 3 : élargissement du remblai et consolidation.

Increase with time of the fill unit weight (thickness of an equivalent fill with constant unit weight).

Phase 1 : Reconsolidation of clay at $70 \mathrm{~g}$ :

Phase 2; Construction of the main fill and consolidation period:

Phase 3: Widening of the fill and consolidation period.

lisant une échelle de temps classique pour les études de consolidation (temps du prototype égal au temps réel du modèle multiplié par le carré de l'échelle de modélisation, soit 4900 dans le cas étudié).

\section{2}

\section{Surpressions interstitielles}

La figure 5 a montre l'évolution au cours du temps des surpressions interstitielles aux nœuds du maillage qui correspondent aux positions des capteurs de pression interstitielle dans le modèle centrifugé. Pour sa part, la figure 5 b présente l'évolution des surpressions interstitielles mesurées par ces capteurs durant l'essai 5. Ces surpressions ont été déduites des pressions mesurées, auxquelles on a enlevé la pression hydrostatique correspondant à leur position initiale dans une nappe stabilisée à la surface du terrain naturel.

D'une manière générale, les surpressions interstitielles calculées suivent l'histoire du chargement du massif d'argile. La construction instantanée des deux remblais principal et adjacent est bien marquée sur ces courbes. Pour leur part, les surpressions déduites des mesures évoluent de façon atypique pendant la première phase de l'essai fle niveau de la nappe n'avait pas été maintenu constant pendant les 400 premiers jours et l'on a assisté à un assèchement du sol sortant des hypothèses de la théorie de la consolidation), puis évoluent de façon classique. Toutefois, le fait qu'elles deviennent négatives en certains points montre que les conditions réelles du modèle ne sont sans doute pas identiques à celles adoptées pour les calculs.

La comparaison des calculs et des mesures montre deux différences:

- les pics initiaux des surpressions interstitielles sont un peu plus élevés dans les calculs de construction des remblais, alors qu'ils sont plus faibles lors de la phase de reconsolidation du sol;

- la dissipation des surpressions interstitielles est plus importante dans les mesures. Toutefois, les conditions aux limites hydrauliques ont probablement varié de façon imprévue en cours d'essai pour faire descendre les surpressions interstitielles au-dessous de leur valeur minimale théorique.

\section{3}

\section{Tassements}

Les tassements de la surface du massif d'argile sont représentés sur la figure 6 (tassements des points de mesure en fonction du temps), sur la figure 8 (tassements des zones non chargées par les remblais) et sur les figures 9 et 10 (coupes transversales du remblai à des instants caractéristiques).

\section{Tasi}

\section{Tassements des points de la surface}

Les tassements calculés et observés (Fig. 6) correspondent assez bien pour les points de mesure situés sous les remblais principal et adjacent (points 3, 7 et 8) et moins bien pour les points situés sous les talus (points 4,5 et 6 ). Le point 9 donne des résultats atypiques, dans la mesure où les mesures indiquent un tassement plus important que pour les autres points du remblai principal alors que le point est plus loin du remblai adjacent.

Les écarts entre courbes calculées et courbes expérimentales traduisent une nouvelle fois la difficulté que présente la modélisation du comportement des sols sous les bords des remblais. Pour le point 4 , il est possible que les perturbations de la première partie de l'essai (défaut d'alimentation en eau du modèle) puissent expliquer les différences ultérieures, mais la distance des calculs et des mesures est plus grande pour le point 5 , sans explication connue.

Si l'on fait abstraction de la première phase du calcul, les calculs sont beaucoup plus proches des mesures, ce qui incite à rechercher une partie des 


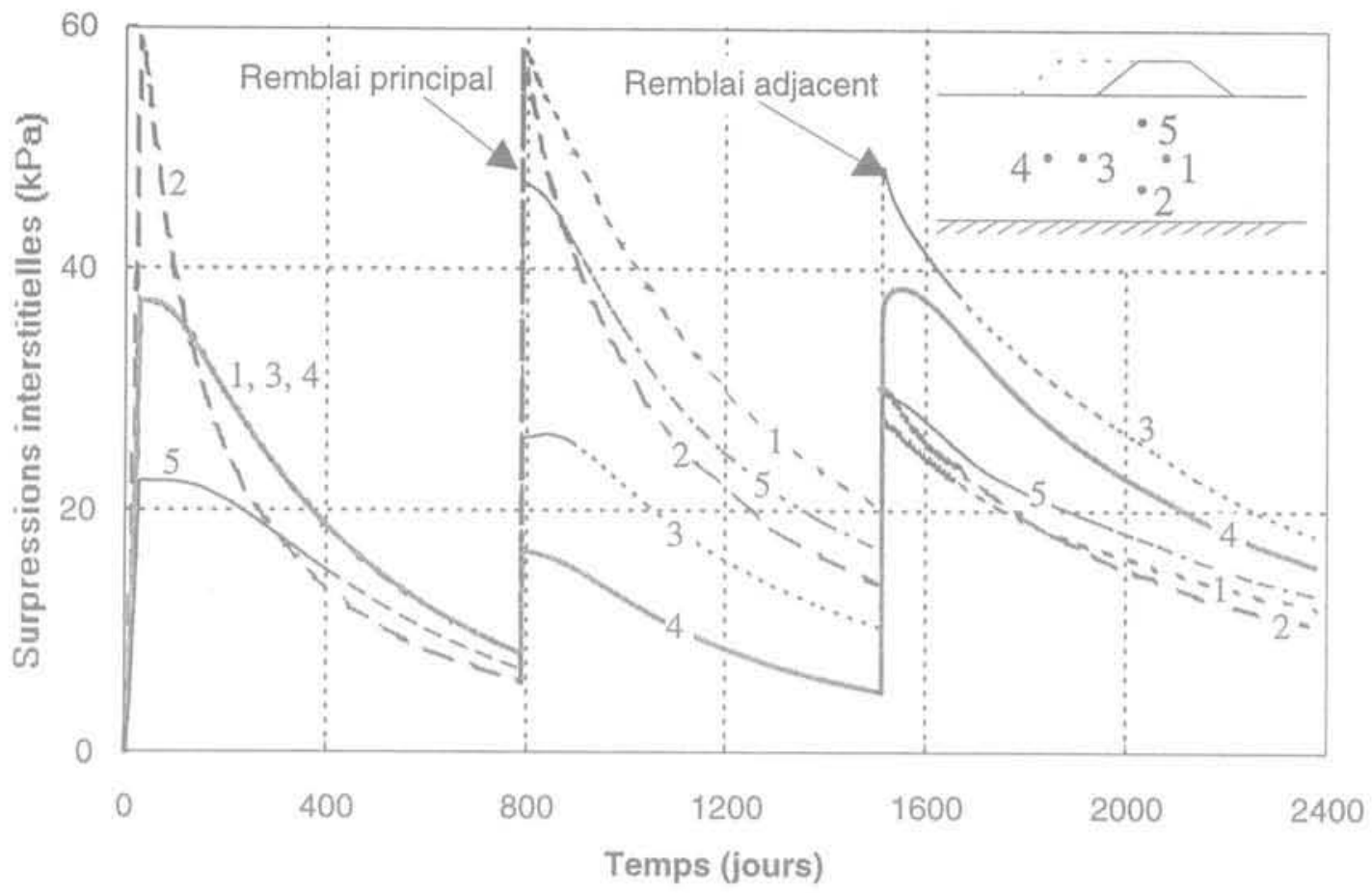

a. Calculs en éléments finis

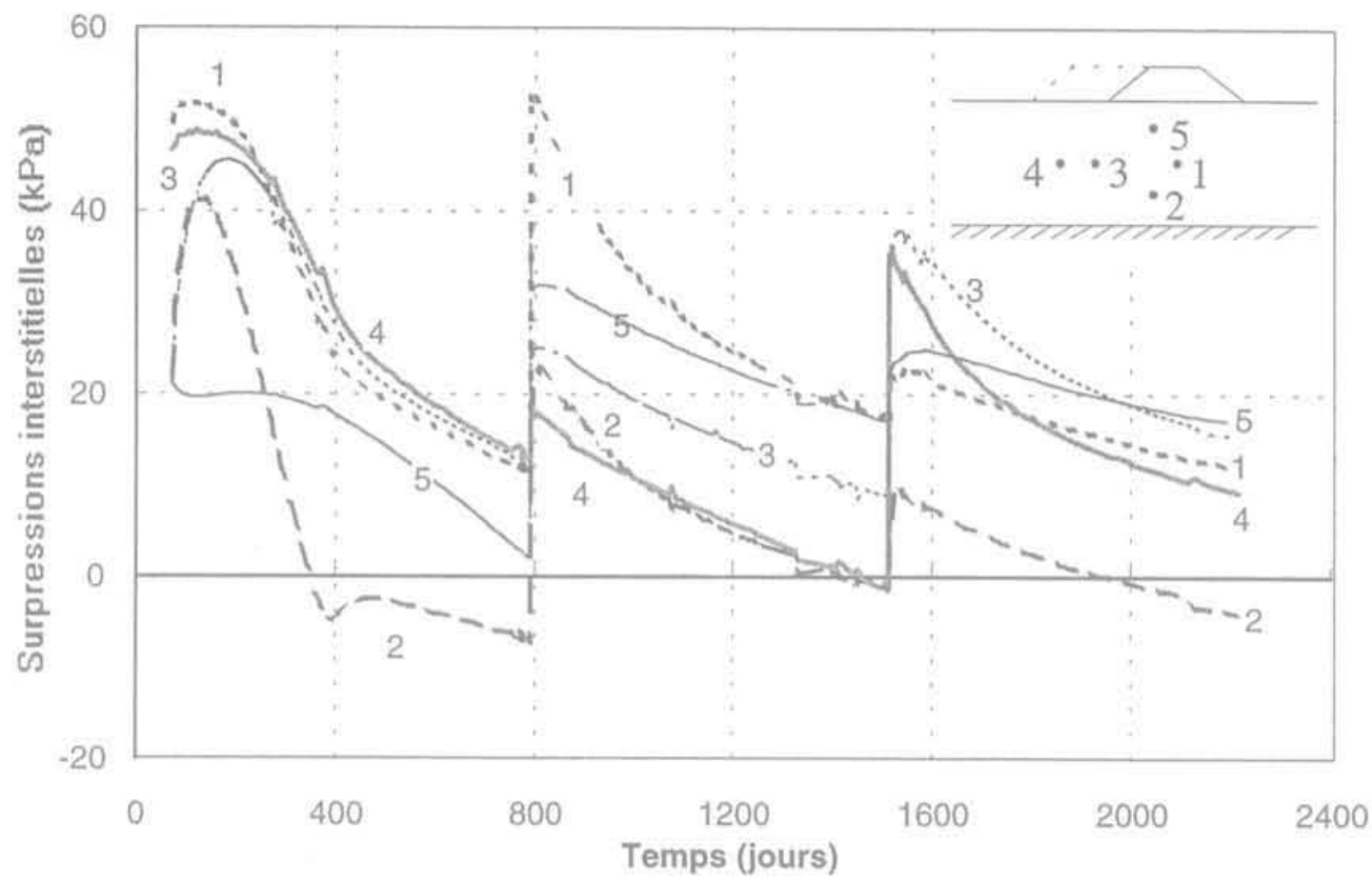

b. Mesures (essai en centrifugeuse)

FG. 5 Evolution au cours du temps des surpressions interstitielles aux points 1 à 5 . a. Calculs en éléments finis. b. Mesures (essai en centrifugeuse).

Changes with time of the excess pore pressures at points 1 to 5 . a. Finite element calculations. b. Measurements (centrifuge test).

causes des divergences dans la modélisation de cette première phase de l'essai. Deux commentaires peuvent être faits :

- la mesure 5 est différente de toutes les autres et il est peu probable qu'elle soit exacte;

- le choix des modules des couches pour la première phase, imposé par le modèle de calcul utilisé lélasticité linéaire anisotrope dans le domaine surconsolidé), reproduit assez mal la courbe de compressibilité semilogarithmique dans sa partie initiale, comme on le voit sur la figure 7. Si l'on avait adopté un module plus fort pour les faibles contraintes, on aurait divisé presque par 10 le coefficient de consolidation du sol et cela aurait pu produire un comportement non drainé plus marqué au voisinage des surfaces drainantes. 


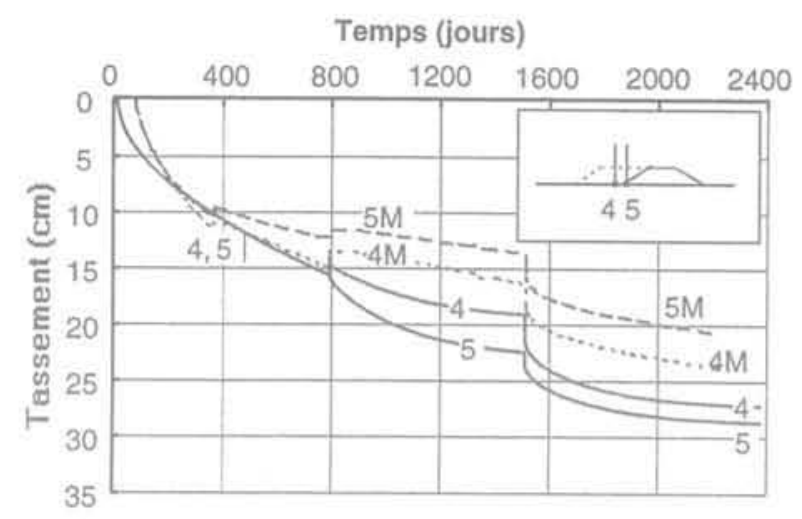

a. Tassements des points 4 et 5

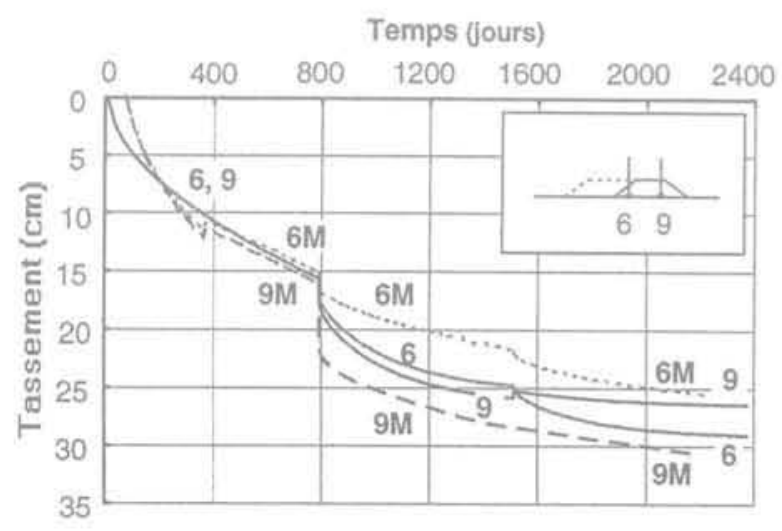

c. Tassements des points 6 et 9

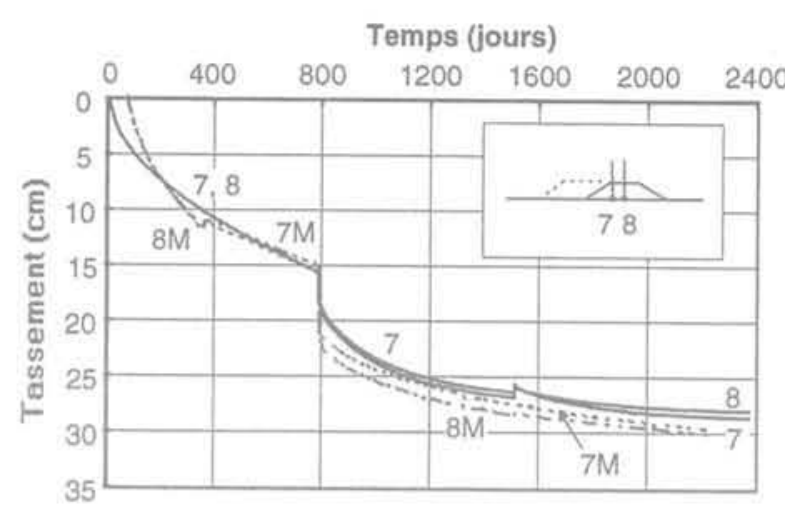

b. Tassements des points 7 et 8

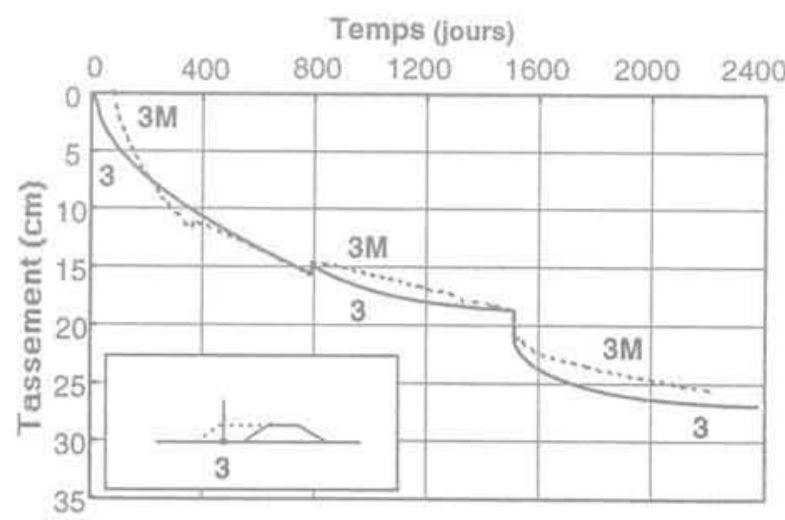

d. Tassement du point 3

FIG.6 Comparaison des tassements calculés et mesurés de la surface de l'argile.

(Les courbes expérimentales, représentées en traits discontinus, sont notées M. Les courbes calculées sont représentées par des traits continus et repérées par des chiffres),

Comparison of calculated and measured settlements of the clay surface (The experimental curves are drawn with dashed or dotted lines and labelled $M$. The calculated curves are drawn with solid lines and referred to using numbers). a. Settlements of points 4 and 5, b. Settlements of points 7 and 8. c. Settlements of points 6 and 9 , d. Settlement of point 3.

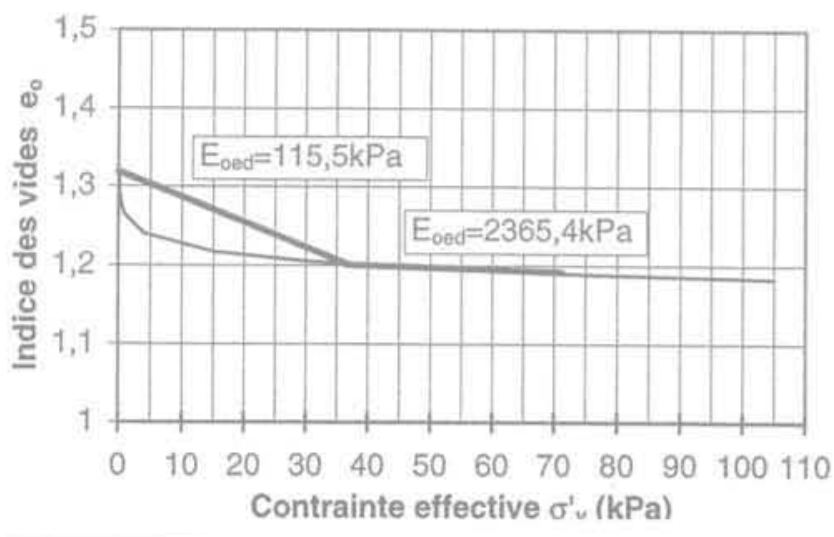

FIG.7 Linéarisation de la courbe œdométrique pour la couche de surface.

Linear approximation of the compressibility curve for the surface layer:

Une autre particularité du calcul, toujours liée à la forme linéaire du modèle et au changement des modules lorsque commence la deuxième phase du calcul, est que le tassement associé aux modules retenus pour la première phase du calcul n'a pas été atteint lorsque l'on est passé à la deuxième étape du chargement, et que la fin de ce tassement a été supprimée par le changement des modules. La première phase de l'essai a été modélisée dans un calcul spécial sans modification des modules pendant 2400 jours pour pouvoir comparer les tassements de l'argile avec les mesures des tassomètres les moins influencés par la construction des remblais (tassomètres 1, 2 et 11). Les courbes de la figure 8 montrent que le calcul surestime le tassement final du massif d'argile, mais sans excès pendant les deux premières phases du calcul. On note, de plus, que la consolidation du sol était loin d'être terminée à la fin de l'essai et que cela a pu avoir des conséquences dans les zones chargées par le remblai.

\section{3,2}

\section{Tassements des remblais selon des coupes transversales}

Les figures 9 et 10 donnent une vue d'ensemble des tassements de la surface du massif d'argile. On retrouve sur ces figures deux phénomènes qui ne sont pas reproduits par le calcul : la poursuite de la consolidation du sol dans la partie non chargée du massif d'argile (capteurs 1, 2 et 11) et la remontée du point 5 . 
Temps (jours)

$04400 \quad 800 \quad 1200 \quad 1600200024002800$

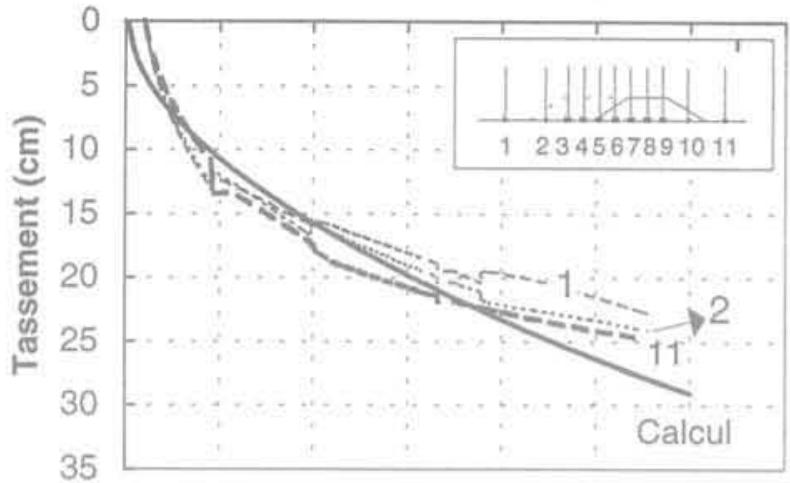

FG. 8 Consolidation de l'argile sans les remblais et comparaison avec les tassements des capteurs 1,2 et 11 .

Consolidation of the clay mass without

embankment loading and comparison with the observed settlements of points 1,2 and 11 .

La poursuite de la consolidation a été commentée ci. dessus. Pour le point 5, on peut noter que sa remontée importante, comme celle de ses voisins, ne peut être reproduite par le calcul, pas plus que l'inclinaison de la droite joignant les tassements des points 7 à 9, qui devrait être horizontale sous le remblai principal, si la charge est uniforme dans la partie centrale du remblai. Il s'agit peut-être du signe d'une amorce de rupture localisée au pied du remblai ou d'une anomalie dans les propriétés du massif d'argile ou encore d'un problème de mesure.

Globalement, les calculs numériques donnent donc une évaluation correcte de l'ordre de grandeur des tas- sements du massif d'argile, mais il subsiste entre les calculs et les mesures des divergences qu'il est difficile d'expliquer et de faire disparaître.

\section{Comparaison des calculs en éléments finis et des méthodes classiques}

Les calculs classiques d'amplitudes de tassements sous les remblais s'effectuent en distinguant :

- un calcul non drainé à court terme, que l'on peut effectuer par exemple avec les abaques de Giroud (1973) pour les charges en forme de remblai ;

- un calcul du tassement final par la méthode cedométrique, qui consiste à calculer la variation des contraintes verticales sur une ligne verticale dans le massif (calcul en élasticité linéaire isotrope, abaque d'Osterberg), puis à en déduire le tassement en utilisant la courbe de compressibilité œedométrique.

Pour ces calculs, nous avons utilisé :

- un module d'Young non drainé uniforme dans toute la couche d'argile $E_{u}=6000 \mathrm{kPa}$ (valeur moyenne dans le massif) et un coefficient de Poisson associé de $v_{u}=$ 0,5

- les paramètres de compressibilité cedométrique qui ont servi à déterminer les paramètres du calcul en éléments finis :

$\mathrm{e}_{\mathrm{o}}=1,316 \mathrm{C}_{\mathrm{s}}=0,04 \quad \mathrm{C}_{\mathrm{c}}=0,5 \quad \sigma_{\mathrm{vo}}^{\prime}=3,7$ à $78,3 \mathrm{kPa}$, avec une pression de préconsolidation supérieure aux contraintes finales (calcul dans le domaine surconsolidé).

Les deux méthodes décrites ci-dessus ont été appliquées successivement au remblai principal (Fig. 11) et au

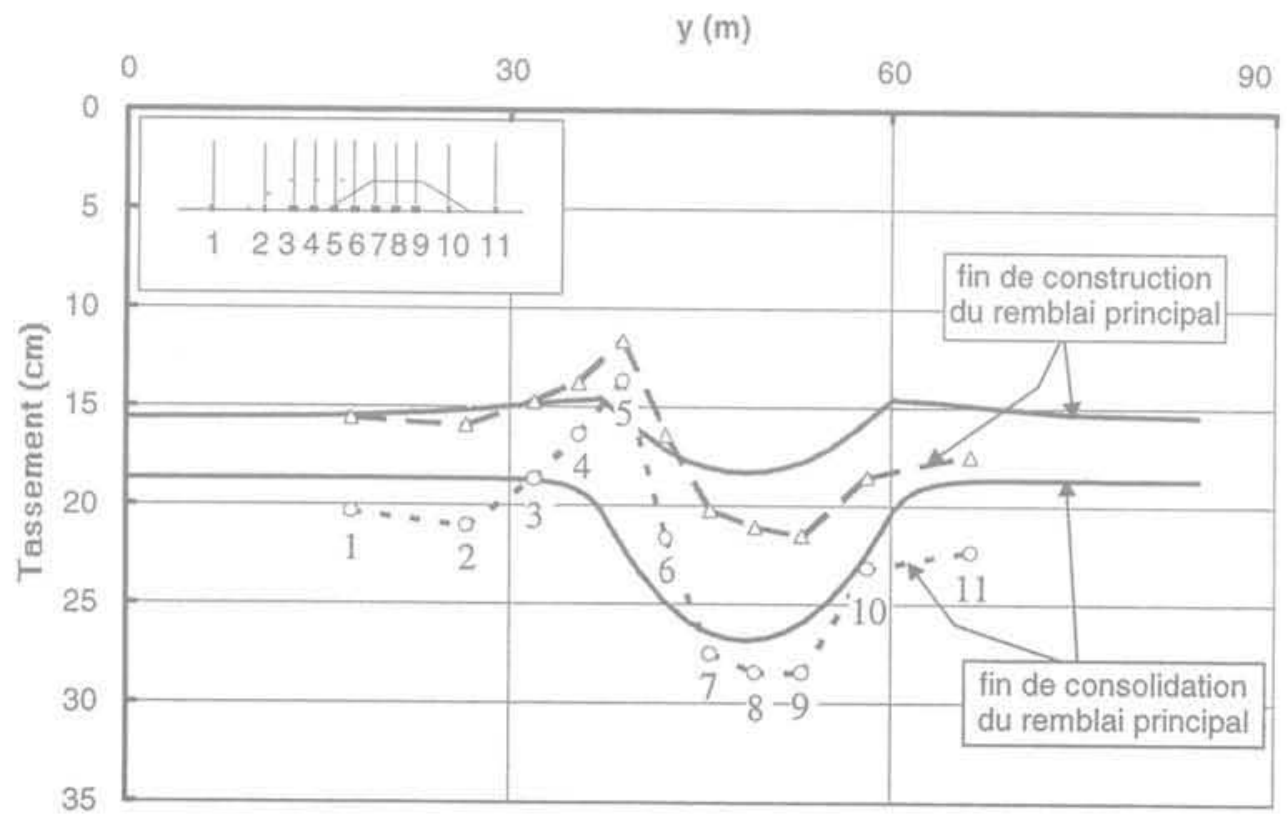

FG.9 Comparaison des tassements calculés (traits continus) et mesurés (traits discontinus) en fin de construction et après consolidation sous l'effet du remblai principal.

Comparison of calculated settlements (continuous lines) and measured

settlements (discontinuous lines) at the end of the construction of the main embankment and after a consolidation period. 


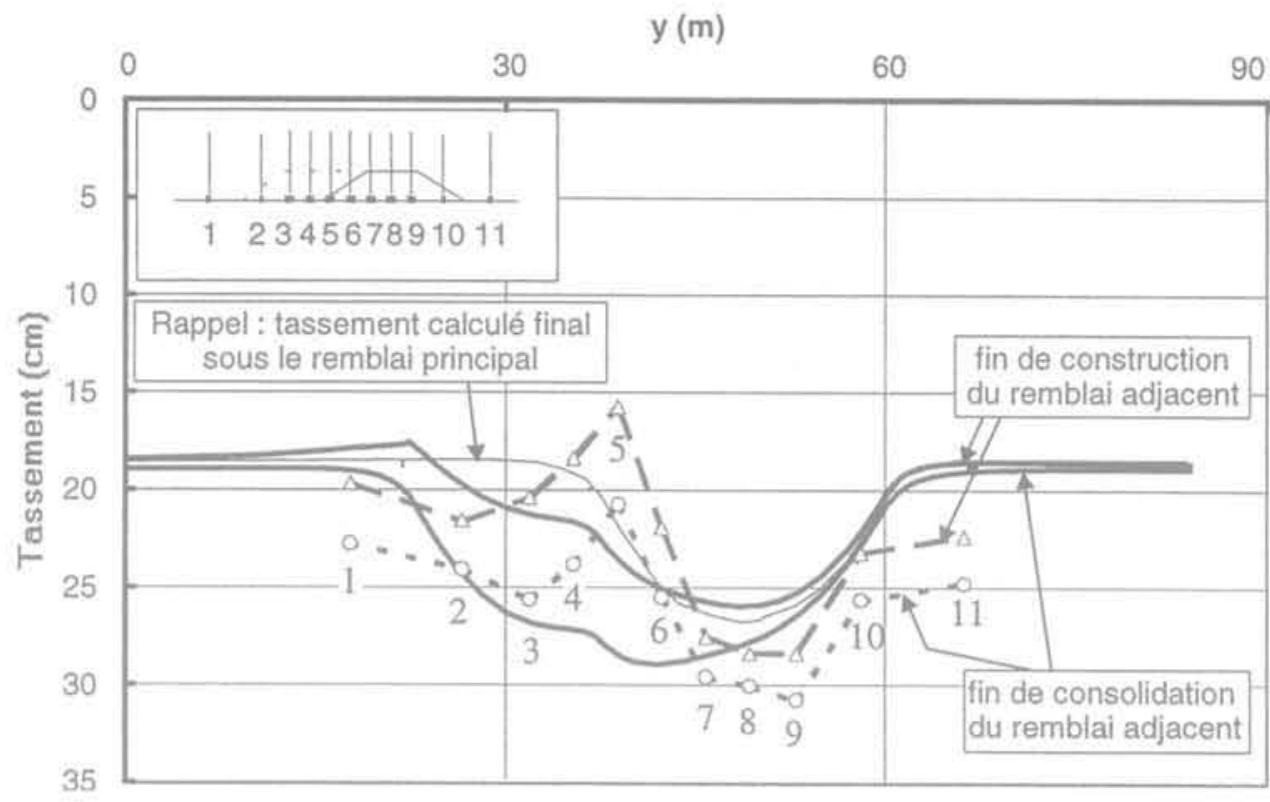

FIG 10 Comparaison des tassements calculés (traits continus) et mesurés (traits discontinus) en fin de construction et après consolidation sous l'effet du remblai adjacent.

Comparison of calculated settlements (continuous lines) and measured

settlements (discontinuous lines) at the end of the construction of the acijacent embankment and after a consolidation period.

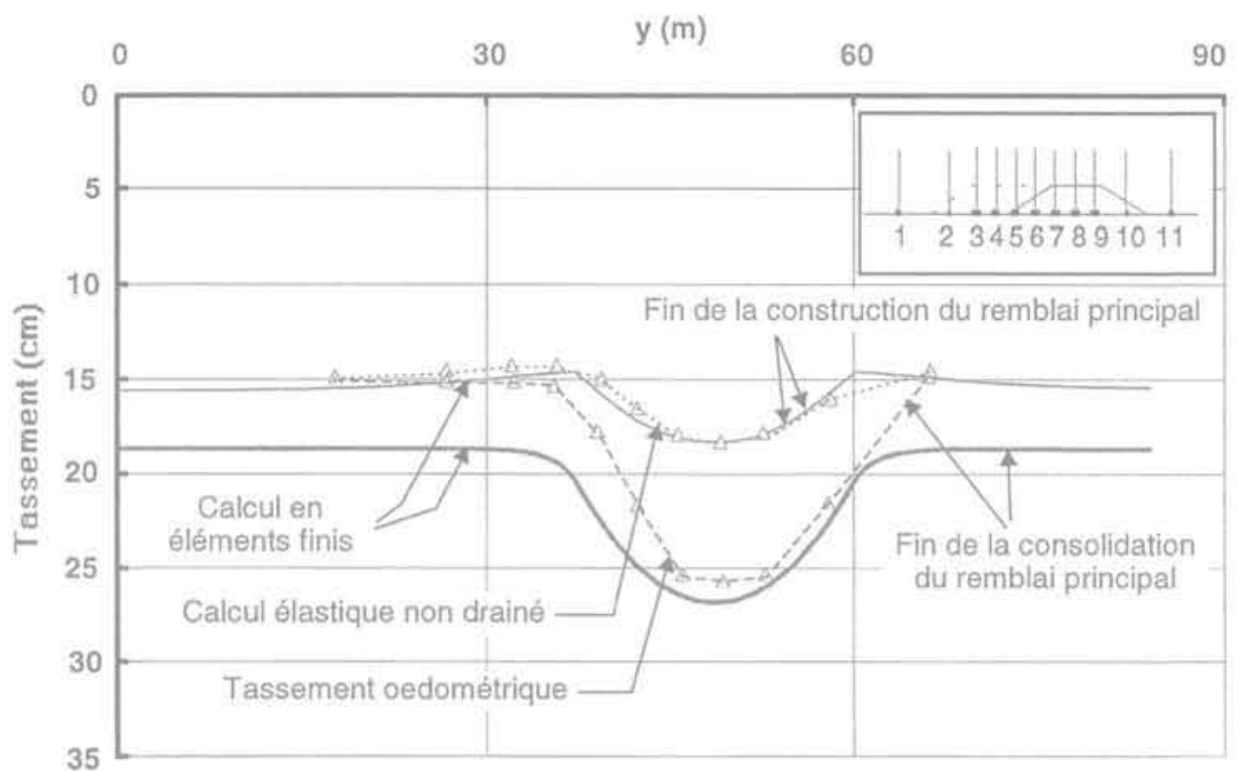

FG, 11 Comparaison des tassements calculés en éléments finis (lignes continues) et par les méthodes classiques (lignes discontinues) pour la construction du remblai principal.

(La courbe de tassement œdométrique représente la somme du tassement non drainé et du tassement différé déduit de la méthode œedométrique).

Comparison of the settlements obtained from the finite element analysis (continuous lines) and from classical methods (discontinuous lines) for the construction of the main embankment. (The a oedometric settlement $x$ curve was obtained by adding the immediate undrained settlement and the differed consolidation settlement estimated by the oedometric method).

remblai adjacent (Fig. 12). Afin de faciliter la comparaison des résultats des calculs, les tassements calculés au moyen de ces paramètres ont été cumulés à partir du tassement calculé en éléments finis à la fin de la première phase de l'essai ( $\mathrm{t}=790$ jours).
On note sur les deux figures que les calculs œedométriques ne prévoient aucun tassement de l'argile en dehors de la zone d'influence des remblais. Cette divergence est compréhensible, puisque les sols naturels ont en général fini leur consolidation quand on vient les 


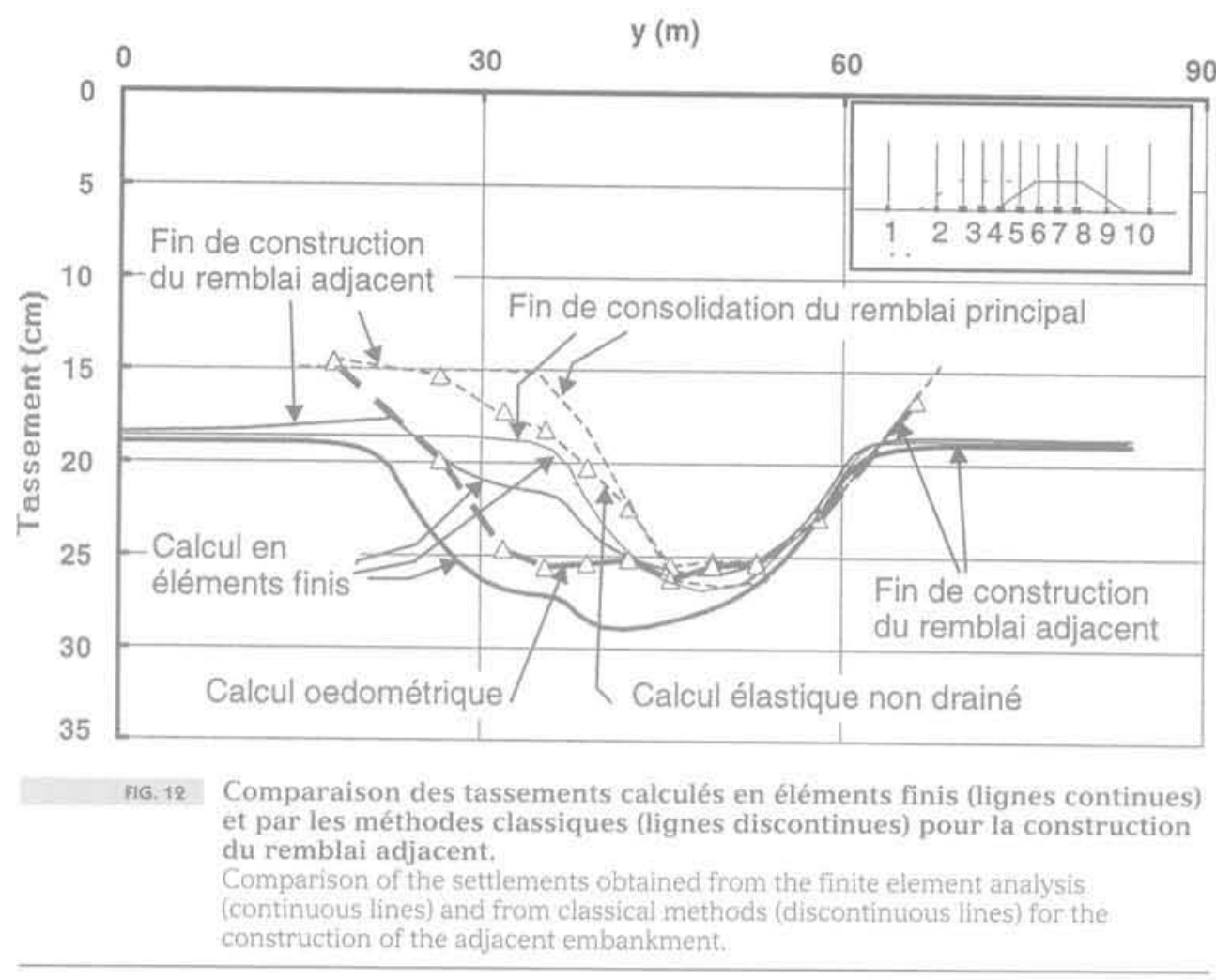

charger par des remblais. Cela rend par contre difficile le calage direct de cette méthode de calcul, reconnue satisfaisante dans la pratique, par analyse d'essais en centrifugeuse sur des modèles de remblais sur argile.

Le tassement calculé en élasticité linéaire (en conditions non drainées) est en assez bon accord avec le calcul en éléments finis dans les deux cas (compte tenu du décalage de l'origine de ces tassements sur la figure 12). Pour les tassements de consolidation, l'écart entre les calculs n'est pas non plus très important et peut être considéré comme associé à la poursuite de la consolidation du sol sous l'état de contrainte appliqué par la rotation de la centrifugeuse.

L'examen des figures $9-10$ et 11-12 permet de comparer les calculs classiques avec les mesures : les écarts, sans être très importants, témoignent aussi du rôle de la consolidation du massif d'argile indépendamment du poids des remblais.

\section{Conclusion}

L'étude présentée dans cet article montre que les mesures effectuées sur ce modèle réduit centrifugé de remblai sur argile sont difficiles à analyser en termes de modèles de calcul : la poursuite de la consolidation du massif d'argile indépendamment de la charge appliquée par les remblais est l'une des causes de cette difficulté. L'absence de mesures de déplacements à l'intérieur du massif d'argile gêne aussi la comparaison détaillée des calculs et du comportement de ce modèle réduit.

Les ordres de grandeur des tassements et pressions interstitielles mesurés sur le modèle ont pu être reproduits de façon satisfaisante en utilisant les paramètres de déformabilité du sol déduits de l'essai œedométrique réalisé en fin d'essai, en admettant que le sol reste à l'état surconsolidé, ce qui est logique compte tenu de la procédure de fabrication du modèle.

Par ailleurs, les méthodes usuelles de calcul des tassements à court terme et différé donnent des résultats du même ordre que les calculs en éléments finis, abstraction faite de la consolidation de l'argile en dehors des remblais.

Néanmoins, l'étude ne permet pas de conclure sur la représentativité par rapport à la nature du modèle de calcul en éléments finis, pas plus d'ailleurs sur celle du modèle réduit: l'interprétation des mesures faites sur des massifs d’argile en centrifugeuse mérite encore des efforts de réflexion, pour la caractérisation des propriétés du massif d'argile et le choix de ses paramètres physiques et mécaniques, en tenant compte de l'interaction de la consolidation et du fluage de l'argile. 


\section{Bibliographie}

Akou Y. - Etude expérimentale et modélisation de l'élargissement des remblais sur sols compressibles, thèse de doctorat de l'Ecole nationale des ponts et chaussées, Paris, 1995, 258 p.

Akou Y. Magnan J.-P. Rault G. - Etude sut modèles de l'élargissement des remblais sur sols compressibles. Laboratoire central des ponts et chaussées, Paris, Etudes et recherches des LPC, série Géotechnique, 1997, 202 p.

Dang M.T., Magnan J.-P. - Application des modèles élastoplastiques de luniversité de Cambridge au calcul du comportement d'ur remblai expérimental sur sols mous. Laboratoire central des ponts et chaussées, rap port de recherche LPC, 1977, $\mathrm{n}^{=} 74,106 \mathrm{p}$
Giroud J.-P. - Tables pour le calcul des fondations. Tome II, Dunod, Paris, 1973. $505 \mathrm{p}$.

Humbert P. CÉSAR-LCPC - Un code général de calcul par éléments finis. Bulletin de liaison des LPC, 1989, $n^{\circ} 160$, pp. 112-115.

Kattan A. - Fluage et consolidation des sols saturés et quasi saturés. Analyse numérique, thèse de doctorat de l'École nationale des ponts et chaussées, Paris, 1990 $199 \mathrm{p}$

Lepidas I., Magnan J.-P. - Fluage et consolidation des sols arcileux : modélisation numérique. Laboratoire central des ponts et chaussées, Paris, rapport de recherche LPC, 1990, $\mathrm{n}^{\circ} 157,167 \mathrm{p}$
Mouratidis A. Magnan J.-P. - Modẻle élastoplastique anisotrope avec écrouissage pour le calcul des ouvrages sur sols compressibles. Laboratoire central des ponts et chaussées, Paris, rapport de recherche LPC, 1983, n²121, $128 \mathrm{p}$.

Piyal M., Magnan J.-P. - Paramètres d'élasticité anisotrope de l'argile molle organique de Cubzac-les-Ponts à l'état surconsolidé. Laboratoire central des ponts et chaussées, Paris, rapport de recherche LPC, 1984, $n^{\circ} 131,108 \mathrm{p}$.

Vermeer P.A., Verruijt A. - An accuracy condition for consolidation by finite elements. International Journal for Numerical and Analytical Methods in Geomechanics, 1991, vol. 5, pp. 1-14. 